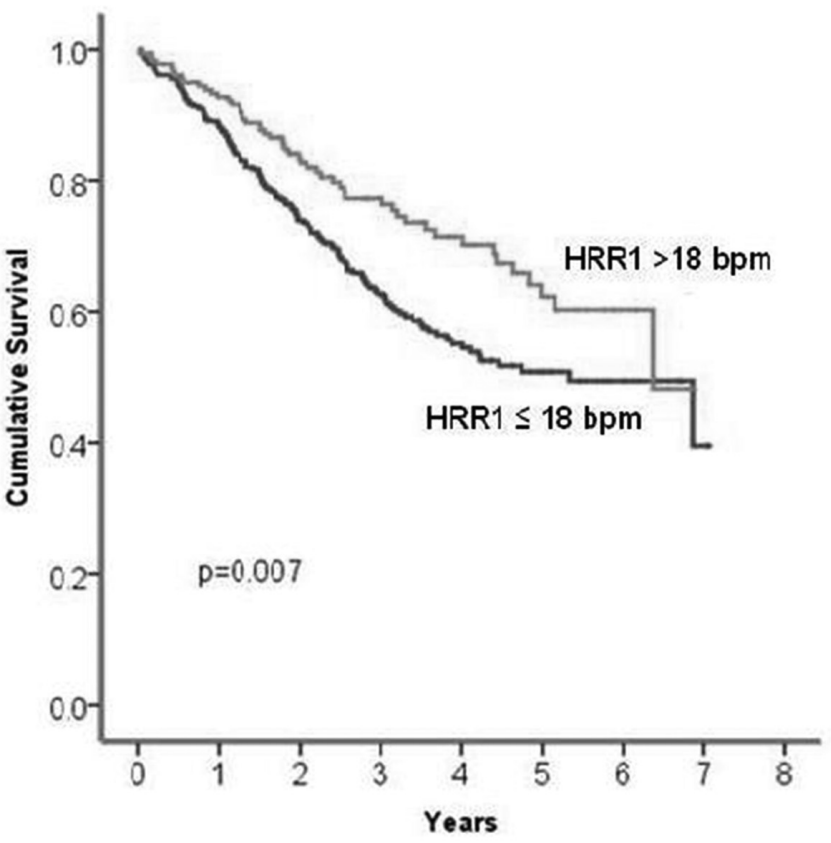

$\begin{array}{llllllll}\leq 18 & 312 & 271 & 199 & 128 & 88 & 44 & 23 \\ >18 & 179 & 161 & 123 & 88 & 58 & 34 & 9 \\ \text { All } & 491 & 441 & 322 & 216 & 146 & 78 & 32\end{array}$

Abstract P170 Figure 1

be a strong predictor of clinical worsening in patients with pulmonary arterial hypertension. ${ }^{1}$ No data as yet has been published regarding the utility of HRR1 as a predictor of mortality.

Aim To assess the prognostic value of HRR1 after an Incremental Shuttle Walk Test (ISWT) in patients with pulmonary hypertension $(\mathrm{PH})$.

Methods Data was retrieved for consecutive cases of $\mathrm{PH}$ diagnosed in our unit from 2001-2010. ISWT was performed routinely as part of baseline assessment according to a modified protocol of Singh et $a .^{2}$ Only treatment-naive patients with ISWT and HRR data from -90 to +30 days from date of diagnosis were included. HRR1 was defined as the difference between maximum heart rate during the ISWT and heart rate after $1 \mathrm{~min}$ of rest following the termination of ISWT.

Results Data was available for 491 patients (including patients from each of the 5 main PH diagnostic groups). HRR1 correlated significantly with ISWT distance, highest heart-rate, maximal change in heart-rate, $\%$ predicted carbon monoxide diffusion (DLco\%pred), pulmonary vascular resistance, cardiac output and mixed venous oxygen saturation ( $\mathrm{p}$ all $\leq 0.01$ ).

Using a cut-off point of $18 \mathrm{bpm}$ the Kaplan Meier graph for survival for patients with HRR1 $>18 \mathrm{bpm}(\mathrm{n}=179)$ was significantly better than HRR $1 \leq 18 \mathrm{bpm}(\mathrm{n}=312), \mathrm{p}=0.007$ (Figure 1) for all patients and for diagnostic Groups 1 and 2 separately $(\mathrm{p}=0.045, \mathrm{p}=0.006)$.

Using univariate Cox proportional hazard analysis for all patients HRR1 (continuous data) had a Hazard Ratio for mortality (HR) of 0.990 with a confidence interval (CI) of $0.962-$ 0.997, $\mathrm{p}=0.008$. Compared to patients with HRR1 $>18$, those with $\mathrm{HRR} 1 \leq 18$ had a HR of 1.559 (CI 1.127-2.156) $\mathrm{p}=$ 0.007 .

Grouping patients by median distance walked and HRR1-18 demonstrated that the HRR1 proved a useful predictor only in patients who walked less than $180 \mathrm{~m}$.
Conclusion HRR1 following ISWT predicts outcome in patients with pulmonary hypertension with more severe disease.

REFERENCES

1 Minai OA et al. Am J Respir Crit Care Med. 2012;185:400-408

2 Singh et al. Thorax 1992;47:1019-1024

\section{P171 IMPLICATIONS OF A NON-CONSTANT RESISTANCE- COMPLIANCE PRODUCT IN PULMONARY ARTERIAL HYPERTENSION}

C Hadinnapola, J Pepke-Zaba, M Toshner. Papworth Hospital NHS Foundation Trust, Cambridge, UK

\subsection{6/thoraxjnl-2014-206260.300}

Introduction Pulmonary vascular resistance (PVR) is traditionally used to describe pulmonary haemodynamic characteristics. However, it does not take into account pulmonary artery compliance (Ca) or pulsatile flow. The product of PVR and $\mathrm{Ca}$ is known as RC-time. RC-time reflects the exponential decay of pressure in the pulmonary arteries during diastole.

The right ventricle (RV) oscillatory power fraction (RVOPF) is the ratio of oscillatory power to total power generated by the RV. Oscillatory power is not used to generate stroke volume. Consequently the RVOPF is a measure of cardiac inefficiency.

Previous small studies have suggested that both RC-time and RVOPF are constant. We hypothesised that RC-time is not constant and changes in RC-time affect cardiac efficiency.

Methods Right heart catheters performed in PapworthHospital over a 6 year period were analysed. Patients were divided into normal pulmonary haemodynamics (NPH) and pulmonary arterial hypertension $(\mathrm{PAH})$ groups. RC-time and RVOPF $(\approx 1-$ (mean pulmonary artery pressure/systolic pulmonary artery pressure) were calculated.

Results RC-time for the NPH group $(0.47 \pm 0.1 \mathrm{~s})$ is lower than the PAH group $(0.56 \pm 0.2 \mathrm{~s} ; \mathrm{p}<0.0001)$. There is an inverse relationship between RC-time and the RVOPF in both groups (PAH group: $\mathrm{r}^{2}=0.33$; NPH group: $\mathrm{r}^{2}=0.27$ ).

Abstract P171 Table 1 Subject characteristics. Data presented as mean $\pm S D$. $p$ - Wilcoxon rank-sum test

\begin{tabular}{|c|c|c|c|}
\hline & NPH group & PAH group & $\mathrm{p}$ \\
\hline $\mathrm{n}$ & 153 & 718 & \\
\hline Age-yrs & $52.6 \pm 13.0$ & $60.4 \pm 14.6$ & $<0.0001$ \\
\hline sPAP-mmHg & $24.3 \pm 3.5$ & $68.2 \pm 21.4$ & $<0.0001$ \\
\hline dPAP-mmHg & $9.7 \pm 2.4$ & $25.3 \pm 8.7$ & $<0.0001$ \\
\hline mPAP-mmHg & $15.9 \pm 2.4$ & $41.1 \pm 12.0$ & $<0.0001$ \\
\hline PCWP-mmHg & $7.7 \pm 2.4$ & $10.5 \pm 2.9$ & $<0.0001$ \\
\hline CO-L.min ${ }^{-1}$ & $4.6 \pm 1.0$ & $4.3 \pm 1.3$ & 0.008 \\
\hline HR-bpm & $74.9 \pm 13.1$ & $78.5 \pm 13.3$ & 0.005 \\
\hline PVR-Wood units & $1.9 \pm 0.5$ & $8.0 \pm 4.8$ & $<0.0001$ \\
\hline Ca-ml.mmHg ${ }^{-1}$ & $4.6 \pm 1.7$ & $1.6 \pm 0.9$ & $<0.0001$ \\
\hline RC time-s & $0.47 \pm 0.1$ & $0.56 \pm 0.2$ & $<0.0001$ \\
\hline RV power total-W & $0.25 \pm 0.07$ & $0.64 \pm 0.24$ & $<0.0001$ \\
\hline $\mathrm{RV}$ power mean- $\mathrm{W}$ & $0.16 \pm 0.05$ & $0.39 \pm 0.14$ & $<0.0001$ \\
\hline RV power oscillatory-W & $0.08 \pm 0.03$ & $0.25 \pm 0.11$ & $<0.0001$ \\
\hline RV oscillatory power fraction & $0.34 \pm 0.08$ & $0.39 \pm 0.06$ & $<0.0001$ \\
\hline
\end{tabular}

NPH group - normal pulmonary haemodynamics group; PAH group - pulmonary arterial hypertension group; SPAP - systolic pulmonary artery pressure; dPAP - diastolic pulmonary artery pressure; mPAP - mean pulmonary artery pressure; PCWP - pulmonary capillary wedge pressure; CO - cardiac output; HR - heart rate; PVR - pulmonary vascular resistance; Ca - pulmonary artery compliance; W - watts 
Conclusions RC-time is not constant between health and pulmonary vascular disease. A reduction in RC-time, in the context of $\mathrm{PAH}$, is associated with a decrease in cardiac efficiency. RVOPF is lower in the NPH group compared to the PAH group. This implies better cardiac efficiency in the NPH group possibly due to less pulsatile loading of the RV. Haemodynamic assessments which include measures of compliance may be of utility in understanding the progression of right heart failure in $\mathrm{PAH}$.

\section{P172 PULMONARY HYPERTENSION IN IPF: UTILITY OF HRCT}

${ }^{1} \mathrm{G}$ Bettini, ${ }^{1} \mathrm{MA}$ Mazzei, ${ }^{1} \mathrm{D}$ Castria, ${ }^{1} \mathrm{E}$ Kacerja, ${ }^{1} \mathrm{RM}$ Refini, ${ }^{2} \mathrm{~F}$ De Negri, ${ }^{1} \mathrm{~N}$ Cioffi Squitieri, 'S Guerrini, 'FG Mazzei, 'P Rottoli, 'L Volterrani. 'Department of Medical, Surgical and Neuro Sciences, University of Siena, Italy., Siena, Italy; ${ }^{2}$ Department of Emergency Medicine, University of Pisa, Pisa, Italy

\subsection{6/thoraxjnl-2014-206260.301}

Purpose To evaluate the reliability of HRCT-parameters suggesting Pulmonary Hipertension $(\mathrm{PH})$ in a population of Idiopathic Pulmonary Fibrosis (IPF) patients with or without $\mathrm{PH}$ at rightsided-heart-catheterization.

Methods and materials The HRCT scans of 26 patients with IPF, underwent both right-sided-heart-catheterization and echocardiography, were retrospectively, blindly and independently, evaluated by one radiology resident and one experienced-radiologist (with a 10-years-experience in thoracic-radiology).

The diameter and area of Pulmonary Artery (PA), before its bifurcation, the diameter of ascending aorta and the mid anteroposterior-diameter of the thoracic vertebra, on the same CTsection, and the widest short-axis-diameters of four segmental arteries and bronchus, in both upper and lower lobes, were measured. The diameters of left ventricle and inferior vena cava, the presence of emphisema, pericardial effusion and hiatal hernia were also evaluated.

Results 15 out of 29 patients had PH at RHC. The HRCT parameters related to the mean pulmonary artery pressure (mPAP) were: the PA-area $\left(\mathrm{R}^{2}=0.238079 ; \mathrm{p}=0.04\right)$, the ratio of the PA-area to the ascending aorta diameter $\left(\mathrm{R}^{2}=0.215345\right.$; $\mathrm{p}$ $=0.0524)$ and above all the ratio of the diameter of segmental artery to the adjacent bronchus in the left upper lobe $\left(\mathrm{R}^{2}=0.354973 ; \mathrm{p}=0.0006\right)$.

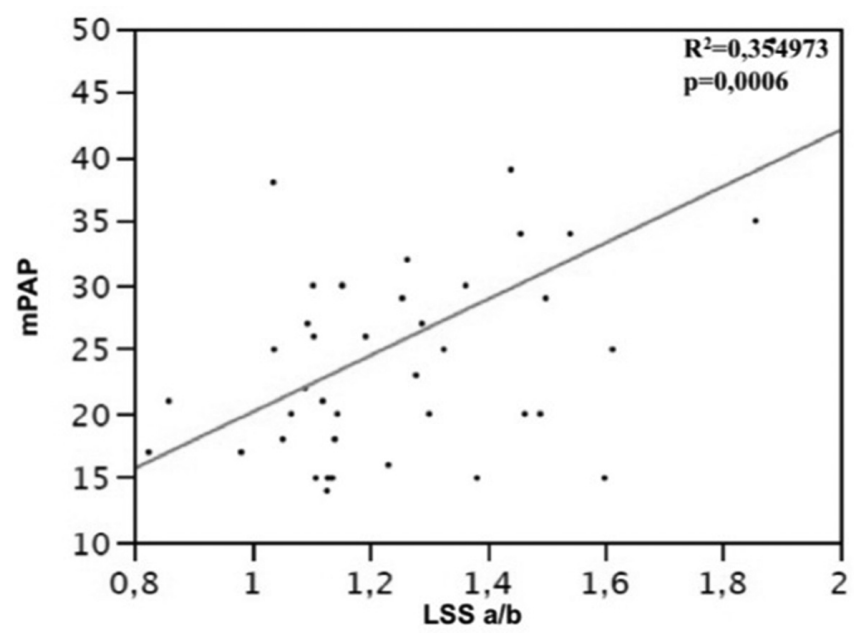

Abstract P172 Figure 1 Linear regression between mean puhnonary artery pressure measured by right heart catheterization (mPAP) and the ratio of the diameter of segmental artery to the adjacent bronchus of the apicoposterior segment of the left upper lobe
The combination of systolic pulmonary artery pressure (sPAP), the PA-area at HRCT and the ratio of the diameter of segmental artery to the adjacent bronchus in the apicoposterior segment of the left upper lobe was strongly correlated to mPAP $\left(\mathrm{R}^{2}=0.785163 ; \mathrm{p}=0.0001\right)$. The contribution of other echocardiographic-parameters (longitudinal STRAIN and Time-to-Peak STRAIN values, TTP) in multivariate regression analysis was not statistically significant, probably because of the small number of patients.

Using the ROC Analysis we found that 931,6 is the upper limit of normal (ULN) for the PA-area, with a $86 \%$ sensitivity and $61 \%$ specificity $(0.839$ AUC); while 20.34 is the ULN for the ratio of the PA-area to the ascending aorta diameter, with a 100\% sensitivity and 50\% specificity (0.804 AUC).

Conclusion (S) HRCT remains an useful tool to identify patients with $\mathrm{PH}$, however the combination of HRCT and echocardiograpy improves accuracy in PH diagnosis.

\section{In the pleural zone}

\section{P173 AMBULATORY MANAGEMENT OF SPONTANEOUS PNEUMOTHORAX}

K Thomas, M Naeem, RV Reddy. Kettering General Hospital, Kettering, UK

\subsection{6/thoraxjnl-2014-206260.302}

Introduction There is no clear consensus on the management of Spontaneous pneumothorax. BTS recommends insertion of chest drain following failure of initial aspiration in large primary spontaneous pneumothorax (PSP) and in all patients with symptomatic/ large secondary spontaneous pneumothorax (SSP). These patients are usually admitted to hospital following chest drain insertion.

Objective To study the feasibility and safety of early discharge of spontaneous pneumothorax patients requiring chest drain on the ambulatory pathway with a Heimlich valve (pneumostat device).

Methods Patients were initially managed as per BTS guidelines. Patients who had a chest drain inserted were admitted until review by the respiratory team. All PSP patients and some SSP patients with good performance status (WHO scale 0-1) were eligible for the ambulatory pathway. Those with continuing air leak are fitted with a Heimlich valve and discharged home. They were reassessed every two days with a CXR on arrival in the ambulatory care unit. The chest drain was removed once the air leak stopped for at least $24 \mathrm{~h}$.

Results 21 episodes of spontaneous pneumothorax in 18 patients (10 PSP and 8 SSP) were treated on the ambulatory pathway between May'13 and June'14. The healthcare usage of patients on ambulatory pathway is listed in the table. The pneumothorax resolved successfully in $82 \%$ (17 episodes). There were three recurrences requiring repeat management on the ambulatory pathway. A total of 10 patients were referred to the surgeon including four with continuing air leak and six due to

\begin{tabular}{lll} 
Abstract P173 Table 1 & & \\
\hline & Range & Average \\
\hline Duration in hospital (days) & $0-4$ & 1.62 \\
Duration in community (days) & $1-12$ & 3.28 \\
Number of reviews & $1-4$ & 1.57 \\
\hline
\end{tabular}

